\title{
Analysis on the Application of Bamboo Environmental Species on Garden Building
}

\section{Dan Zhou, Haicheng Zhao}

Shandong Business Institute, Yantai, Shandong, 264003

Keywords: bamboo environmental species; garden building; application study

\begin{abstract}
Bamboo has become one of the most popular design materials in the 21st century. As a modern building material made of biomass materials with many advantages, it has high social, economic and ecological benefits. At the same time, bamboo has a high aesthetic value since ancient times, and its shape has gathered elegant and beautiful charm. Therefore, bamboo materials have increasingly become a building material sought after by major designers.

In modern gardens, garden buildings with bamboo as materials have become increasingly popular. As more and more ornamental bamboo species have been successfully domesticated and introduced in the northern region, the application of ornamental bamboo in urban landscaping has been opened up. In addition to extensive planting in parks and streets, many types of ornamental bamboo can also be used for potted plants and bonsai applications. Through extensive experimentation and screening, based on the full study of bamboo biology, the application of domesticated ornamental bamboo in gardens is explored.
\end{abstract}

\section{The Origin and Development of Bamboo in Landscape Architecture}

Zhou first used bamboo in the royal gardens to record: "The emperor's western expedition, as for the mysterious pool, the forest of the tree, is the bamboo forest." ("Mu Tianzi Biography") This is the earliest Chinese record of bamboo application. Later, Qin Shihuang introduced the "Shanglinyuan" to Yungang Suzhu to Xianyang cultivation. Since then, the Northern Wei Dynasty, the Tang Dynasty, the Southern Song Dynasty, the Ming and Qing Dynasties and other successive generations have applied bamboo. Among them, Wang Wei's design is more famous. Zhujing, such as "Jianzhuling" and "Bamboo Hall” in the "Suichuan Other Industry". These examples are typical records of bamboo in ancient Chinese gardens.

Today, bamboo has become one of the most popular design materials of the 21st century. As a modern building material made of biomass materials with many advantages, it has high social, economic and ecological benefits. At the same time, bamboo has a high aesthetic value since ancient times, and its shape has gathered elegant and beautiful charm. Therefore, bamboo materials have increasingly become a building material sought after by major designers. The distribution of bamboo resources around the world is uneven, and the industrial development of various countries is uneven. At the same time, the level of scientific and technological development in various regions is also uneven.

The East pays attention to traditional industries and the West focuses on emerging industries. The East pays attention to human elements, and the West pays attention to environmental elements and design. The bamboo industry in most developing countries in Asia is in its infancy, and China is in a period of transition from traditional to modern development. For European and American countries, they use bamboo as a new resource mainly in emerging fields such as service industry and energy development. Even though the bamboo production in Europe and the United States is small, they have fully applied bamboo in the construction industry, landscaping and ecological protection through the development of bamboo biological properties. 


\section{Bamboo Design Principles in Modern Gardens}

As an environmentally friendly material in gardens and buildings, bamboo materials not only require technological advancement, but also the advancement of artistic beauty. Therefore, through the hands of designers, bamboo materials present architectural beauty and beauty. Therefore, the architectural structure is actually the embodiment of the designer's thoughts and radiance. The aesthetic sense based on the physical structure can only be called a successful work.

The texture of bamboo is very evident in the architecture. For example, the 2010 Shanghai World Expo is a new form of garden landscape. The Vietnam Pavilion uses bamboo as the main building material of the exhibition hall. The bamboo rattan weaving is used as the building skin to bring out the beauty of the bamboo texture. This is manifested by the external characteristics of the bamboo skin, which is an important part of the use of texture in the design of bamboo materials. Similarly, the combination of bamboo and other materials is a deep sense of the layer of culture and culture, so that the design is not limited to the single expression of the external texture. Bamboo has the characteristics of order aesthetics that exist in music. The ancients made bamboo into a musical instrument, such as bamboo, bamboo flute, etc., to play beautiful music. Similarly, in modern gardens and architecture, the musical characteristics of bamboo are also promoted, and the artistic integration into the bamboo design has become a different kind of wonderful work by the designer's hand. When a large number of bamboos are piled up together, it is disorderly and unorderly, but after careful design by the designer, it has achieved the order beauty of bamboo, which is the unique beauty given by the aesthetics of order. Since ancient times, bamboo has been given a gentleman with a high spirit and a bright and elegant character. This kind of gentleman's temperament has become the spiritual symbol of the Chinese nation and has become a valuable asset of Chinese history and culture. In the long history of history, the poetry of bamboo has attracted countless literati and singers to sing. Therefore, China's strong bamboo culture has played a huge role in promoting the emergence and development of Chinese bamboo art. The bamboo cultural works inherited in Chinese classical gardens are widely loved by the West and are highly respected by the Western royal family. Many Western royal gardens also have many bamboo cultural works that imitate Chinese classical gardens. This is an embodiment of the artistic conception that is irreplaceable by Chinese traditional bamboo culture. Even with the continuous development of modern science and technology, the artistic conception of traditional bamboo culture has continued, reflected in the ever-changing modern design. Bamboo has a systematic principle in garden architecture, mainly through step-by-step construction steps, such as design concept, assembly method, structure and other steps, to produce bamboo components and materials according to the established modulus and size, thus becoming A strict constitution system. Systematic development will greatly promote the industrialization of the bamboo industry and promote the application and development of bamboo materials in gardens and buildings. Environmental protection, green, recycling and sustainability are the characteristics we look at when selecting bamboo. It is also because of the biological advantages of bamboo that it has become the first choice for modern materials. At the same time, when using bamboo, the natural growth cycle should be strictly followed, and excessive harvesting should be prohibited. Secondly, when using bamboo, ecological utilization should be the dominant factor, pay attention to recycling, and make the best use of it.

\section{The Application of Bamboo in Garden Building}

At present, there are only wood structure specifications in China's structural specifications, and there is no independent bamboo structure specification. Only some experimental norms of domestic and foreign architects and architects can be referenced. Due to the lack of relevant research, the type, structure and construction process of bamboo garden buildings are not well known to designers. Based on the experience of some garden bamboo construction in China and related literatures at home and abroad, the different uses of bamboo in landscape architecture are now described.

The large round bamboo has high compressive strength and can be used as a foundation column 
for concrete construction or as a foundation column for concrete slabs. It is generally used in garden buildings for 1 or 2 storey buildings. According to actual needs, a plurality of small diameter round bamboos can be bundled into columns, which are buried in the soil after anti-corrosion treatment to replace the concrete columns. Unpreserved bamboo is highly perishable in the soil. It is usually treated with anti-corrosion treatment or embedded in a gas-tight material such as concrete to extend its service life. Bamboo columns should be selected for dry humidity and bamboo of the same diameter and thin section to obtain maximum compressive strength.

In China, many bamboos are tied into bundles for garden architecture. They are mainly composed of round bamboo or long bamboo, bundled with bamboo, hemp rope or iron wire. In order to enhance the rigidity, more bamboo and wood are mixed. Due to the size and spacing of the bamboo, the bamboo building frame is less rigid and not as good as the wood structure, but its anti-side shear and resilience properties are superior, and its seismic resistance is strong, which is especially suitable for earthquake areas. For example, 20 sets of bamboo structure safety classrooms donated by Hunan University in Beijie Primary School of Guangyuan City, Sichuan Province have been installed and formed, and are deeply loved by people in the earthquake zone.

It is very common to use bamboo as a scaffold for garden construction. Bamboo scaffolding can be adjusted and expanded according to the shape of the building. The key is that it is attached to the basic structure of the building. With this support structure, bamboo scaffolding has room for expansion in the vertical direction. The structure is generally double-row bamboo rafts, tied with hemp rope or iron wire, mainly composed of vertical poles, diagonal poles, top supports and large poles. Each layer is used as a scaffolding board for transportation. Bamboo scaffolding is mostly used for exterior construction of garden buildings, and can also be used for residential buildings and temporary buildings. Its low cost and strong wind resistance, but due to the large variability of bamboo diameter size, it is not suitable for high-rise. Some construction management departments have now specified that bamboo scaffolding is only allowed for construction of houses below 5 floors.

Bamboo is a good roofing material in garden architecture because of its light weight, high strength, flexibility and impact resistance. 1) Half back bamboo tile roof. Take the round bamboo from the middle to the second, to the section; after the air is dried in the shade; the semi-circular bamboos are embedded in the front and back, and then drilled and nailed to the sandalwood strip (Figure 1). 2) Bamboo roofing. The bamboo pieces are mostly taken from large-diameter round bamboos and made into bamboo pieces with a width of 2 to $4 \mathrm{~cm}$. They are arranged side by side or staggered, and the nails are nailed on the sandalwood strips. 3) Bamboo mat tile roof. This is a kind of bamboo building material developed by the Academy of Forestry in Sichuan Province in recent years. It is made of bamboo sassafras and is woven into a mat. It is glued and hot pressed on a metal wave mold. It has the characteristics of large format and construction. 4) After the bamboo decoration roof is completed, various bamboo roofs are installed on it to achieve the effect of the bamboo roof on the appearance. In addition, roofs are covered with reeds, bamboo branches, straw, etc. on the roof support. Although this kind of roof is a traditional method, it seems to be simple, but it is easy to draw, simple in construction, low in price, coordinated with bamboo, and has good landscape effect. It is also more common in modern gardens.

Bamboo can also be used as a small span or medium span garden bridge for car dealers or pedestrians. For example, a bamboo cable woven into a rope shape with bamboo rafts can be used as a suspension bridge instead of a steel wire rope. The bamboo cable bridge spanning the Dujiangyan River in the west of Sichuan Province in China is $340 \mathrm{~m}$ long and is also known as the "narrow bridge". It is a well-known bamboo cable bridge in the world. Bamboo reinforced concrete is made of ordinary Portland cement as the condensing material, with polystyrene foam, rice husk and orange rod as light aggregate, starch modified rubber as intermediate binder and calcium chloride as early strength agent. Using sodium alkyl sulfonate as a mixing aid, bamboo ribs are used as lightweight aggregate concrete for the skeleton of lightweight wallboard. The utility model has a certain elastic deformation amount, and the outer wall does not need to use the heat insulation layer, which effectively reduces the dust pollution, saves the cost, and improves the building efficiency 
and the earthquake resistance. Bamboo reinforced concrete is mainly used for lightweight walls. It is widely used in garden engineering. It can be used as an empty exterior wall for frame structures or used in conjunction with other buildings. It can also be used alone, such as as a wall and a manhole cover. The tensile strength of bamboo tendons can reach $80 \%$ of steel bars, and the tensile strength of bitter bamboos with a growth age of about 4 years is estimated to be $50 \%$ of steel bars, but its modulus of elasticity is only $1 / 10$ of that of steel bars. Therefore, it is not feasible to replace the steel bars with bamboo ribs. However, due to the low requirements of the garden buildings, the prospects for their use are worth looking forward to.

\section{Conclusion}

As a modern garden and building material, bamboo has an important role in the world's green development in terms of bamboo culture and biological characteristics. In the face of the deteriorating ecological environment, the pursuit of green ecology, environmental and environmental protection, the global forest area of tropical rain forests has shrunk dramatically, and under the background of over-exploitation of wood resources, bamboo materials have excellent characteristics and are getting more and more The more widely used.

\section{Acknowledgements}

Fund Project: 2018 Shandong Province Art Science Key Project "Innovative Application Research of Natural Bamboo Materials in Environmental Pieces"

\section{References}

[1] He Yunxiao, Zhou Sijian, Zhao Yinquan, Gan Tingjiang. Application of Bamboo in Chinese Garden [J].Journal of Anhui Agricultural Sciences, 2007(18):28.

[2] Dou Ying, Yu Xuejun. Development and Comparison of World Bamboo Industry [J]. World Agriculture, 2008 (7): 12.

[3] Huang Zubo, Li Chunbao, Wu Yan, et al. Application of bamboo in civil engineering [J]. 2007, 23(2): 79 - 80.

[4] Jiang Zehui, Wang Zheng, Chang Liang, et al. Research on manufacturing technology of bamboo wall for building [J]. Journal of Beijing Forestry University, 2006, 28(6): 155 - 158.

[5] Zhu Ziqiang. Talking about bamboo and bamboo reinforced concrete [J]. Jiangxi Building Materials, 2007 (3): 6 - 18. 